\title{
TRATAMENTO DAS OSTEOMIELITES CRÔNICAS
}

\author{
José Wagner de Barros, Constantino Jorge Calapodopulos, Décio José \\ Oliveira e Paulo Miki Júnior
}

\begin{abstract}
Entre 1986 e 1990, sessenta pacientes com osteomielite crônica foram tratados cirúrgica e quimioterapicamente sendo reavaliados, tanto no aspecto clínico como radiológico, num espaço de tempo de doze a sessenta meses. O tratamento cirúrgico foi associado à quimioterapia por sulfametoxazol e trimetoprim, por via oral, durante seis meses. O resultado foi considerado bom quando houve desaparecimento dos sinais e sintomas do processo infeccioso. Isto ocorreu em 50 (83,33\%) pacientes submetidos a uma única intervenção cirúrgica e em 9 (15\%) submetidos a mais de uma cirurgia. Em um $(1,67 \%)$ caso a osteomielite não foi debelada.
\end{abstract}

Palavras-chaves: Osteomielite. Osteomielite crônica. Quimioterapia em osteomielite. Tratamento das osteomielites crônicas. Tratamento cirúrgico das osteomielites.

As osteomielites crônicas ocorrem, na maioria dos casos, através de complicações das fraturas expostas, de contaminação cirúrgica (osteomielite pós-traumática) apresentando com grande freqi ència em nosso meio, em decorrência do elevado número de acidentes com máquinas motorizadas e do crescente número de cirurgias ortopédicas. A persistência do processo infeccioso da osteomielite hematogênica aguda é outra maneira dessas afecções cronificarem. Neste caso a infecção óssea aguda ocorre através da via sanguínea, com embolização de foco infeccioso a distância, comum em pacientes de baixo poder sócio-econômico ${ }^{13} 18$. Nestas infecções, a necrose óssea associada pode servir como meio de cultura para bactérias que não se multiplicam rapidamente, mas persistem em latência.

Entre 1986 e 1990, submetemos a tratamento cirúrgico e quimioterápico um grupo de pacientes com osteomielite crônica (OC) e nos pareceu justificada a publicação dos resultados obtidos.

\section{MATERIAL E MÉTODOS}

Na Tabela 1, encontram-se os principais dados de identifícação dos pacientes. Antes de qualquer

\footnotetext{
Disciplina de Ortopedia e Traumatologia da Faculdade de Medicina do Triângulo Mineiro (FMTM), Uberaba, MG. Endereço para correspondência: Dr. José Wagner de Barros. Disciplina de Ortopedia e Traumatologia/HE/FMTM, Av. Getúlio Guaritá 130, 38025-440 Uberaba, MG.

Recebido para publicação em 17/08/92.
}

medida terapêutica os pacientes foram radiografados em duas posições (frente e perfil). No local da fístula foi colhido material da secreção para o exame bacteriológico e antibiograma. O primeiro constituiu de cultura dos germes presentes na secreção e, para o antibiograma, testou-se a resistência da bactéria frente aos antibióticos.

A todos os pacientes ministrou-se de rotina, 4 comprimidos diários de sulfametoxazol $(1600 \mathrm{mg}) \mathrm{e}$ trimetoprim (310 mg) (co-trimoxazole). Paralelamente, utilizou-se outros antimicrobianos (oxacilina, cloranfenicol, gentamicina, cefalotina), sempre que o resultado do antibiograma não confirmasse suscetibilidade ao sulfametoxazol + trimetoprim. Simultaneamente, ou no máximo uma semana após o início da terapêutica medicamentosa, o paciente foi submetido à cirurgia, que se iniciou com a fistulografia com azul de metileno. A seguir realizou-se ampla exposição do ferimento, tendo sido excisados, a pele cronicamente infectada e os tecidos moles e músculos mal vascularizados. $\mathrm{Na}$ etapa seguinte removeram-se os tecidos ósseos necrosados e infectados até serem encontradas estruturas sangrantes. A saucerização foi realizada quando possível; a estabilização da não união com fixador externo e enxertia óssea autóloga ${ }^{16}$ foram efetuados quando necessários. A pele não foi suturada e a ferida deixada aberta, permitindo a cicatrização por segunda intenção.

O pós-operatório constou de curativos diários com solução fisiológica e polivinilpirrolidona iodo. Após a granulação, a pele foi suturada apenas nos pacientes em que a ferida cirúrgica não se encontrava 
Barros JW, Calapodopulos CJ, Oliveira DJ, Miki Júnior P. Tratamento das osteomielites crônicas. Revista da Sociedade Brasileira de Medicina Tropical 25:235-239, out-dez, 1992.

Tabela 1 - Dados gerais de sessenta pacientes, etiologia, tipo, tratamento e evolução da osteomielite crônica.

\begin{tabular}{|c|c|c|c|c|c|c|c|c|}
\hline $\begin{array}{c}\text { Caso } \\
\mathrm{N}^{0} \\
\end{array}$ & $\begin{array}{l}\text { Idade } \\
\text { (anos) }\end{array}$ & Sexo & Localização & Etiologia & Tipo & $\begin{array}{c}\text { Internação } \\
\text { (dias) }\end{array}$ & $\begin{array}{c}\text { Seguimento } \\
\text { (meses) }\end{array}$ & Resultado \\
\hline 1 & 18 & $\mathrm{~F}$ & tibia & S. aureus & pós-traumática & 20 & 20 & curado \\
\hline 2 & 36 & M & tíbia & S. aureus & pós-traumática & 13 & 12 & curado \\
\hline 3 & 33 & $\mathrm{M}$ & tíbia & $P$. aeruginosa & pós-traumática & 64 & 14 & curado \\
\hline 4 & 65 & M & tíbia & P. aeruginosa & pós-traumática & 23 & 20 & curado \\
\hline 5 & 19 & $\mathrm{M}$ & fềmur & S. aureus & hematogênica & 45 & 21 & curado \\
\hline 6 & 24 & M & tíbia & S. aureus & pós-traumática & 30 & 19 & curado \\
\hline 7 & 11 & $\mathbf{M}$ & úmero & S. aureus & hematogênica & 27 & 23 & curado \\
\hline 8 & 53 & $\mathrm{~F}$ & fềmur & S. aureus & pós-traumática & 10 & 16 & curado \\
\hline 9 & 58 & $\mathbf{M}$ & fềmur & S. aureus & pós-traumática & 51 & 38 & curado \\
\hline 10 & 60 & $\mathbf{M}$ & fềmur & S. aureus & pós-traumática & 14 & 49 & curado \\
\hline 11 & 35 & $\mathrm{~F}$ & tíbia & S. aureus & pós-traumática & 18 & 20 & curado \\
\hline 12 & 51 & $\mathbf{M}$ & rádio & S. aureus & pós-traumática & 46 & 51 & curado \\
\hline 13 & 13 & M & fềmur & S. aureus & hematogênica & 8 & 21 & curado \\
\hline 14 & 21 & $\mathrm{~F}$ & fềmur & S. aureus & hematogênica & 27 & 56 & curado \\
\hline 15 & 42 & $\mathbf{M}$ & fêmur & S. aureus & pós-traumática & 24 & 45 & curado \\
\hline 16 & 67 & $\mathrm{M}$ & fềmur & $P$. aeruginosa & pós-traumática & 74 & 38 & curado \\
\hline 17 & 53 & $\mathbf{M}$ & fềmur & P. mirabilis & pós-traumática & 37 & 50 & curado \\
\hline 18 & 46 & $\mathrm{M}$ & rádio & S. aureus & hematogênica & 76 & 20 & curado \\
\hline 19 & 25 & $\mathrm{M}$ & tíbia & S. aureus & pós-traumática & 8 & 18 & curado \\
\hline 20 & 46 & $\mathrm{M}$ & úmero & S. aureus & pós-traumática & 36 & 23 & curado \\
\hline 21 & 65 & $\mathrm{~F}$ & fêmur & S. aureus & pós-traumática & 90 & 22 & curado \\
\hline 22 & 25 & $\mathrm{~F}$ & púbis & S. aureus & hematogênica & 11 & 17 & curado \\
\hline 23 & 24 & $\mathbf{M}$ & tibia & S. aureus & pós-traumática & 33 & 21 & curado \\
\hline 24 & 12 & $\mathrm{~F}$ & tíbia & S. aureus & hematogênica & 24 & 23 & curado \\
\hline 25 & 7 & $\mathrm{~F}$ & fêmur & S. aureus & hematogênica & 33 & 22 & curado \\
\hline 26 & 29 & $\mathbf{M}$ & calcâneo & S. aureus & hematogênica & 22 & 17 & curado \\
\hline 27 & 44 & $\mathbf{F}$ & tíbia & S. aureus & pós-traumática & 30 & 21 & curado \\
\hline 28 & 33 & $\mathbf{M}$ & ísquio & S. aureus & hematogênica & 13 & 20 & curado \\
\hline 29 & 42 & $\mathbf{M}$ & tíbia & S. aureus & hematogênica & 18 & 21 & curado \\
\hline 30 & 25 & $\mathbf{M}$ & fêmur & E. coli & pós-traumática & 18 & 26 & curado \\
\hline 31 & 77 & $\mathbf{F}$ & fềmur & S. aureus & pós-traumática & 41 & 53 & curado \\
\hline 32 & 38 & $\mathbf{M}$ & ulna & S. aureus & pós-traumática & 20 & 57 & curado \\
\hline 33 & 27 & $\mathbf{M}$ & rádio & S. aureus & pós-traumática & 9 & 40 & curado \\
\hline 34 & 72 & $\mathbf{F}$ & fêmur & E. coli & pós-traumática & 63 & 46 & curado \\
\hline 35 & 37 & $\mathbf{M}$ & rádio & S. aureus & pós-traumática & 19 & 41 & curado \\
\hline 36 & 23 & $\mathbf{F}$ & fềmur & S. aureus & pós-traumática & 47 & 20 & curado \\
\hline 37 & 19 & $\mathrm{M}$ & fêmur & S. aureus & hematogênica & 15 & 33 & curado \\
\hline 38 & 85 & $\mathbf{F}$ & fêmur & S. aureus & pós-traumática & 42 & 49 & curado \\
\hline 39 & 16 & $\mathbf{M}$ & tarso & S. aureus & hematogênica & 14 & 30 & curado \\
\hline 40 & 46 & $\mathbf{M}$ & ilíaco & P. mirabilis & pós-traumática & 24 & 27 & curado \\
\hline 41 & 60 & $\mathbf{M}$ & fềmur & S. aureus & pós-traumática & 37 & 56 & curado \\
\hline 42 & 32 & $\mathbf{M}$ & tíbia & S. aureus & hematogênica & 30 & 20 & curado \\
\hline 43 & 24 & $\mathrm{M}$ & tíbia & S. aureus & pós-traumática & 30 & 18 & curado \\
\hline 44 & 9 & $\mathrm{~F}$ & fềmur & S. aureus & hematogênica & 23 & 17 & curado \\
\hline 45 & 46 & $\mathbf{M}$ & fềmur & S. aureus & pós-traumática & 15 & 55 & curado \\
\hline 46 & . 18 & $F$ & fêmur & S. aureus & hematogênica & 22 & 28 & curado \\
\hline 47 & 42 & $\mathbf{M}$ & tíbia & S. aureus & hematogênica & 18 & 21 & curado \\
\hline 48 & 19 & $\mathbf{M}$ & fềmur & S. aureus & pós-traumática & 18 & 52 & curado \\
\hline 49 & 57 & $\mathbf{M}$ & fềmur & S. aureus & pós-traumática & 5 & 48 & curado \\
\hline 50 & 15 & $\mathbf{F}$ & tíbia & S. aureus & hematogênica & 9 & 19 & curado \\
\hline $51 *$ & 27 & $\mathbf{M}$ & parietal & S. aureus & pós-traumática & 43 & 16 & curado \\
\hline $52 *$ & 41 & $\mathrm{~F}$ & fềmur & S. aureus & pós-traumática & 44 & 25 & curado \\
\hline $53 *$ & 41 & $\mathbf{M}$ & fêmur & S. aureus & pós-traumática & 28 & 35 & curado \\
\hline $54 *$ & 27 & M & tíbia & S. aureus & hematogênica & 24 & 20 & curado \\
\hline $55 *$ & 34 & M & fềmur & S. aureus & hematogênica & 30 & 18 & curado \\
\hline $56 *$ & 28 & $\mathbf{M}$ & tíbia & S. aureus & pós-traumática & 18 & 18 & curado \\
\hline $57 *$ & 30 & M & tíbia & S. aureus & pós-traumática & 35 & 30 & curado \\
\hline $58 *$ & 8 & F & fêmur & S. aureus & hematogência & 22 & 18 & curado \\
\hline $59 *$ & 62 & $\mathbf{M}$ & fềmur & S. aureus & pós-traumática & 22 & 34 & curado \\
\hline $60 *$ & 37 & $\mathbf{F}$ & fêmur & S. aureus & pós-traumática & 22 & 51 & recidiva \\
\hline
\end{tabular}

* mais de uma intervenção cirúrgica 
Barros JW, Calapodopulos CJ, Oliveira DJ, Miki Júnior P. Tratamento das osteomielites crônicas. Revista da Sociedade Brasileira de Medicina Tropical 25:235-239, out-dez, 1992.

totalmente obliterada. Os doentes continuaram a quimioterapia com sulfametoxazol + trimetoprim, na mesma dosagem já referida, após a alta hospitalar. O outro antibiótico, quando utilizado, foi administrado só no período de internação.

No domicílio, os pacientes continuaram a fazer uso do quimioterápico, perfazendo um período total de 6 meses, tendo sido orientados quanto à importância da manutenção regular da medicação. Esta era entregue após alta hospitalar e nas supervisões ambulatoriais realizadas a cada 15 dias.

Uma segunda cirurgia foi indicada quando os pacientes apresentaram recidiva do processo infeccioso e o tratamento cirúrgico, nestes casos, foi semelhante ao realizado na primeira intervenção.

Os resultados foram considerados bons quando os sinais e sintomas da infecção desapareceram.

\section{RESULTADOS}

Em 39 (65\%) dos 60 pacientes a OC foi póstraumática e em 21 (35\%) hematogênica (Tabela 1). Em $29(48,3 \%)$ casos, o processo localizou-se no fêmur, em 18 (30\%) na tíbia e no restante $(21,3 \%)$ em outros ossos do esqueleto. O agente etiológico foi o Staphilococcus aureus em 53 (88,3\%) casos; em $3(5 \%)$ a $P$. aeruginosa; em $2(3,3 \%)$ a $P$. mirabilis; e em $2(3,3 \%)$ a E. coli (Tabela 1$)$.

O tempo de internação foi em média de 29 dias (mínimo de 5 e máximo de 90). O seguimento ambulatorial foi no mínimo de 12 meses e no máximo de 57 meses (Tabela 1).

Dos 60 pacientes, $50(83,33 \%)$ curaram-se após a quimioterapia e uma única cirurgia (Tabela 1). A cicatrização da ferida ocorreu, em média, com 28 dias. Dos 10 pacientes restantes, 9 tiveram de submeter a duas ou mais cirurgias e todos também se curaram. Em um paciente (caso $n^{\circ} 60$ ) o processo infeccioso-inflamatório não respondeu à terapêutica empregada.

Os pacientes que utilizaram outro quimioterápico além do co-trimoxazole encontramse na Tabela 2.

\section{DISCUSSÃO}

O tratamento adequado das osteomielites crônicas é discutível. A maioria dos autores acha que é necessário manter níveis altos de antimicrobianos no tecido infectado por período prolongado, administrando antibiótico parenteral por 1 a 2 meses seguido por um outro período por via oral. A recomendação acima tem poucos dados

Tabela 2 - Pacientes que utilizaram outro antibiótico além do co-trimoxazole.

\begin{tabular}{|c|c|c|c|c|c|c|}
\hline $\begin{array}{c}\text { Caso } \\
\text { No }^{\circ}\end{array}$ & $\begin{array}{l}\text { Idade } \\
\text { (anos) }\end{array}$ & Sexo & Localização & Etiologia & Tipo & Antibiótico \\
\hline 1 & 18 & $\mathrm{~F}$ & tíbia & S. aureus & pós-traumática & oxacilina \\
\hline 2 & 36 & $\mathrm{M}$ & tibia & S. aureus & pós-traumática & cefalotina \\
\hline 3 & 33 & $\mathbf{M}$ & tíbia & $P$. aeruginosa & pós-traumática & cefalotina \\
\hline 4 & 65 & $\mathbf{M}$ & tíbia & $P$. aeruginosa & pós-traumática & gentamicina \\
\hline 5 & 19 & $\mathrm{M}$ & fêmur & $S$. aureus & hematogênica & gentamicina \\
\hline 6 & 24 & $\mathbf{M}$ & tíbia & S. aureus & pós-traumática & gentamicina \\
\hline 7 & 11 & $\mathrm{M}$ & úmero & S. aureus & hematogênica & gentamicina \\
\hline 8 & 53 & $\mathrm{~F}$ & fêmur & S. aureus & pós-traumática & gentamicina \\
\hline 9 & 58 & $\mathbf{M}$ & fêmur & $S$. aureus & pós-traumática & gentamicina \\
\hline 10 & 60 & $\mathbf{M}$ & fêmur & S. aureus & pós-traumática & gentamicina \\
\hline 11 & 35 & $\mathrm{~F}$ & tíbia & $S$. aureus & pós-traumática & cloranfenicol \\
\hline 12 & 51 & $\mathbf{M}$ & rádio & S. aureus & pós-traumática & cloranfenicol \\
\hline 13 & 13 & M & fêmur & S. aureus & hematogênica & oxacilina \\
\hline 14 & 21 & $\mathrm{~F}$ & fêmur & $S$. aureus & hematogênica & oxacilina \\
\hline 15 & 42 & $\mathbf{M}$ & fêmur & S. aureus & pós-traumática & oxacilina \\
\hline 16 & 67 & $\mathbf{M}$ & fêmur & $P$. aeruginosa & pós-traumática & oxacilina \\
\hline 17 & 53 & $\mathrm{M}$ & fêmur & P. mirabilis & pós-traumática & oxacilina \\
\hline 18 & 46 & $\mathrm{M}$ & rádio & S. aureus & hematogênica & oxacilina \\
\hline $51 *$ & 27 & $\mathbf{M}$ & parietal & $S$. aureus & pós-traumática & oxacilina \\
\hline $54 *$ & 27 & M & tibia & S. aureus & hematogênica & oxacilina \\
\hline $57 *$ & 30 & $\mathbf{M}$ & tíbia & $S$. aureus & pós-traumática & cefalotina \\
\hline $60 *$ & 37 & $\mathrm{~F}$ & fêmur & $S$ aureus & pós-traumática & oxacilina \\
\hline
\end{tabular}

* mais de uma intervenção cirúrgica 
Barros JW, Calapodopulos CJ, Oliveira DJ, Miki Júnior P. Tratamento das osteomielites crônicas. Revista da Sociedade Brasileira de Medicina Tropical 25:235-239, out-dez, 1992.

relevantes para suportá-la e geralmente se aplica à osteomielite estafilocócica ${ }^{15}$, mas é pouco apreciável no caso das osteomielites crônicas causadas por certos organismos gram-negativos.

Os dados mais expressivos da utilização de quimioterápicos vêm dos estudos de Bell ${ }^{2}$, que usou cloxacilina VO em doses de $5 \mathrm{~g}$ mais probenecida em dose de $2 \mathrm{~g} / \mathrm{dia}$. Os pacientes foram tratados por um mínimo de 6 meses e alguns até 1 ano. $O$ seguimento contínuo de 19 pacientes por 7 a 9 anos mostrou bons resultados, isto é, fechamento da fístula e ausência de recorrências clínicas. Kelly e cols ${ }^{11}$ trataram 425 pacientes com osteomielite crônica, submetidos a tratamento cirúrgico e antimicrobiano por 4 semanas e obtiveram um índice de cura de $84,4 \%$.

Nos casos, por nós estudados, a cura clínica do processo infeccioso ocorreu após uma única intervenção cirúrgica em $50(83,33 \%)$ pacientes e em $9(15 \%)$ com mais de uma, utilizando a quimioterapia por 6 meses.

Escolhemos o trimetoprim associado ao sulfametoxazol por seu espectro de ação sobre o Streptococcus, Staphylococcus e Enterobacter ${ }^{4} 9$ 1017 , podendo eventualmente ser ativos com a Pseudomonas aeruginosa ${ }^{12}$, além de serem bem tolerados quando utilizados por tempo prolongado ${ }^{1}$ 71214 . A combinação do produto trimetoprim com sulfametoxazol inibe a síntese do ácido nucléico da bactéria por dois mecanismos. O componente sulfonamida inibe a sintese do ácido deidrofólico da bactéria pela competição com o ácido paraaminobenzóico. $\mathrm{O}$ componente trimetoprim inibe reversivelmente a enzima deidrofolate reductase. Esta é responsável pela redução do ácido deidrofólico a tetraidrofolato, fonte de energia utilizada pela bactéria na sua reprodução ${ }^{4910}$.

Vários relatos referem que a terapia antimicrobiana por tempo prolongado possa ser uma abordagem promissora no tratamento dessas afecções. Contudo, não temos dados comprobatórios em nosso estudo para afirmar que os resultados obtidos são devidos somente ao uso do quimioterápico. O desbridamento cirúrgico adequado é fundamental no tratamento dessas infecções, e a cura por segunda intenção, utilizada como parte deste protocolo, parece ter sido fator muito importante na obtenção destes resultados além da longa permanência hospitalar.

\section{SUMMARY}

From 1986 to 1990, sixty patients were treated for chronic osteomyelitis with surgery and chemotherapy. The clinical and radiologic follow-ups ranged from twelve to sixty months. The surgery was associated with a six-month oral sulfamethoxazole-trimethoprim combination chemotherapy. Clinical cure was achieved when inflammatory signs and symptoms disappeared, as happened to 50 (83.33\%) of the single surgery cases, and to 9 (15\%) of the multiple surgery cases. For one case (1.67\%) osteomyelitis was not cured.

Key-words: Osteomylitis. Chronic osteomyelitis. Chemotherapy for osteomyelitis. Chronic osteomylitis treatment. Surgery for osteomyelitis.

\section{AGRADECIMENTO}

Agradecemos ao Prof. José Tavares-Neto o auxílio prestado no planejamento deste estudo.

\section{REFERÊNCIAS BIBLIOGRÁFICAS}

1. Barr $A L$, Whineray $M$. Immune thrombocytopenia induced by cotrimoxazole. Austrie Journal Medical 10:54-55, 1980.

2. Bell S. Further observations on the value of oral penicillin on chronic staphylococcal osteomyelitis. Austrie Medical Journal 2:591-593, 1976.

3. Black J, Hunt TL, Godley PJ. Oral antimicrobial therapy for adults with osteomyelitis or septic arthritis. Journal Infectious Disease 155:968-972, 1987.

4. Burchall JJ. Mechanism of action of trimethoprimsulfamethoxazole. II. Journal Infectious Disease 128:437-441, 1973.

5. Bushdy SRM. Trimethoprim-sulfamethoxazole: $I n$ vitro microbiological aspects. Journal Infectious Disease 128: 442-462, 1973.

6. Dukes MNG. Meyler's side effects of drugs. Elsevier, Amsterdam, 1988.

7. Dunkle LM, Brock N. Long-term follow-up of ambulatory management of osteomyelitis. Clinics Pediatrics 21:650-654, 1982.

8. Eisenberg J, Kitz D. Savings from out patient antibiotic therapy for osteomyelitis. Economic analysis of a therapeutic strategy. The Journal of the Americam Medical Association 255:1584-1588, 1986.

9. Foltzer MA, Reese RE. Trimethoprimsulfamethoxazole and other sulfonamides. Medical Clinies North America 71:1177-1194, 1987.

10. Hitchings GH. Mechanism of action of trimethoprimsulfamethoxazole. I. Journal Infectious Disease 
Barros JW, Calapodopulos CJ, Oliveira DJ, Miki Júnior P. Tratamento das osteomielites crônicas. Revista da Sociedade Brasileira de Medicina Tropical 25:235-239, out-dez, 1992.

128:433-436, 1973.

11. Kelly PJ, Fitzgerald $J_{\Gamma} R$. Results of treatment of tibial and femoral osteomyelitis in adults. Clinical Orthopaedics and Related Research 259:295-303, 1990.

12. Kucers A, Bennett NM. The use of antibiotics, JB Lippincott Company, Philadelphia, 1987.

13. Lemos C, Raso P. Ossos e articulações. In: Lopes ER, Chapadeiro E, Raso P, Tafuri WL (ed). Bogliolo Patologia. 4.edição. Guanabara Koogan, Rio de Janeiro p.812-854, 1987.

14. McEvoy GK. AHFS drug information 1990. Bethesda. American Society of Hospital Pharmacists, 1990.
15. Norden CW. Osteomyelitis. In: Mandel GL, Douglas RG, Benett JE (ed). Principles and practice of infectious disease. 3. edição. Churchill Livingstone, London p.922-930, 1990.

16. Papineau LJ, Alfageme A, Delcourt IP, Pilon L. Osteomyelitis chronique: excision et greffe de spongieux a l'air libre apres mises a plat extensives. International Orthopedie 3:165-176, 1979.

17. Stiefelod SM, Graziani AL, MacGregor RR, Esterhai JL. Toxicities of antimicrobial agents used to treat osteomyelitis. Orthopedic Clinics North America 22:439-465, 1991.

18. Volpon JB. Osteomielite. Revista Brasileira Clínica Terapêutica 13:258-263, 1984. 